\title{
Complete Minimal Residual Disease Response
}

National Cancer Institute

\section{Source}

National Cancer Institute. Complete Minimal Residual Disease Response. NCI Thesaurus.

Code C124427.

Absence of residual disease based on pre-defined thresholds and MRD neg ativity by laboratory techniques. 